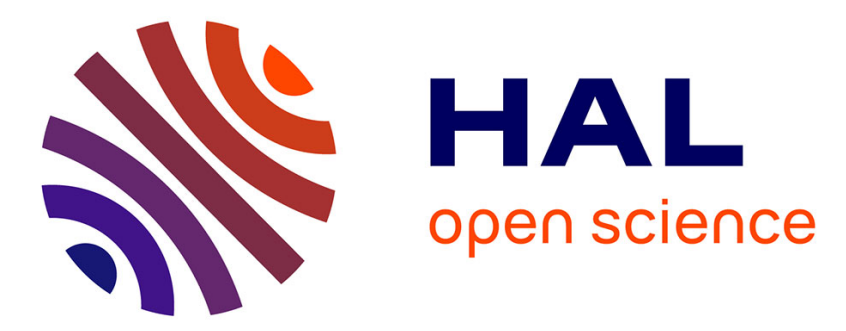

\title{
Les matériaux supraconducteurs : problèmes et perspectives d'utilisation. Nouvelles orientations des recherches
}

Jean Etourneau

\section{- To cite this version:}

Jean Etourneau. Les matériaux supraconducteurs: problèmes et perspectives d'utilisation. Nouvelles orientations des recherches. Revue de Physique Appliquée, 1986, 21 (11), pp.649-657. 10.1051/rphysap:019860021011064900 . jpa-00245484

\section{HAL Id: jpa-00245484 https://hal.science/jpa-00245484}

Submitted on 1 Jan 1986

HAL is a multi-disciplinary open access archive for the deposit and dissemination of scientific research documents, whether they are published or not. The documents may come from teaching and research institutions in France or abroad, or from public or private research centers.
L'archive ouverte pluridisciplinaire HAL, est destinée au dépôt et à la diffusion de documents scientifiques de niveau recherche, publiés ou non, émanant des établissements d'enseignement et de recherche français ou étrangers, des laboratoires publics ou privés. 


\title{
Les matériaux supraconducteurs : problèmes et perspectives d'utilisation. Nouvelles orientations des recherches
}

\author{
J. Etourneau \\ Laboratoire de Chimie du Solide du CNRS, Université de Bordeaux I, 351, cours de la Libération, \\ 33405 Talence Cedex, France
}

(Reçu le 18 mars 1986, révisé le 26 mai, accepté le 27 mai 1986)

\begin{abstract}
Résumé. - Les matériaux supraconducteurs déjà utilisés ou en voie de développement dans l'industrie (applications en courant fort et en microélectronique) font l'objet d'une étude critique : les alliages $\mathrm{Nb}$-Ti et les composés A-15. Les problèmes et les perspectives d'utilisation des phases de Chevrel sont abordés. On montre que les idées nouvelles et les phénomènes nouveaux résultent des études conduites dans quatre secteurs : (1) coexistence magnétisme-supraconductivité (supraconductivité de parois dans les ferromagnétiques, supraconductivité induite par le champ magnétique, (2) recherche de la supraconductivité de type p ou triplet (composés à fermions lourds et composés ferromagnétiques itinérants), (3) supraconductivité et onde de densité de charge dans les composés de basse dimensionalité, (4) les supraconducteurs organiques.
\end{abstract}

Abstract. - In a first part of this review are summarized advances in superconducting materials concerning their developments in both research and industry (conductors for superconducting magnets, Josephson junction devices in microelectronics) : Nb-Ti solid solution alloy and A-15 type compounds. The problem of Chevrel phases is discussed in view of their application in high field superconducting magnets. In a second part it is shown that the study of superconducting materials is producing a stream of new ideas in different ways : (1) interplay of superconductivity and magnetism : superconducting Bloch-wall in ferromagnets ; magnetic field induced superconductivity, (2) p-wave or triplet superconductivity : heavy fermion superconductors, itinerant ferromagnetic superconductors, (3) interplay of superconductivity and charge density wave, (4) organic superconductors.

\section{Introduction}

La supraconductivité sous tous ses aspects fondamentaux et appliqués ne peut se développer que grâce au caractère interdisciplinaire des recherches. Pour juger les possibilités d'améliorer les propriétés supraconductrices des matériaux, il faut se rappeler que même si la théorie microscopique de la supraconductivité, proposée par Bardeen, Cooper et Schrieffer (BCS) en 1957 a connu un grand succès en interprétant et formulant les phénomènes, le problème par exemple du calcul de la température critique $\left(T_{\mathrm{cr}}\right)$ d'un matériau donné demeure encore aujourd'hui très difficile. Cette difficulté provient du fait que la supraconductivité dépend de détails fins dans la structure électronique, qui ne peuvent être saisis quantitativement dans des matériaux complexes. Une théorie des supraconducteurs par opposition à une théorie de la supraconductivité devrait débuter par des prédictions a priori de la structure cristalline, ce que personne ne sait faire jusqu'à présent. Il faut donc souligner que les résultats obtenus dans la recherche de matériaux supraconducteurs (hautes températures critiques, propriétés de fermions lourds...) n'ont été bien souvent que le fait du hasard ou bien ont découlé d'une approche empirique à travers des observations à caractère cristallographique, atomique et métallurgique dans des séries de matériaux.

Deux préoccupations doivent motiver les recherches : (1) améliorer les matériaux existants, tant pour ceux qui sont utilisés pour les applications que pour ceux qui sont le support d'études fondamentales (2) préparer de nouveaux composés (formes métastables de matériaux existants, compositions et structures nouvelles) pour mieux comprendre la supraconductivité et tenter d'élever $T_{\mathrm{cr}}$, pour rechercher d'autres types de couplages électron-électron autre que celui faisant intervenir les phonons, pour étudier l'interaction de la supraconductivité avec le magnétisme ou les ondes de densité de charges par exemple.

Après quelques rappels généraux sur les supraconducteurs une étude critique sera faite concernant les matériaux déjà utilisés ou en voie de développement dans l'industrie (applications en courant fort et en microélectronique); problèmes de mise en forme (multifilaments, rubans, couches minces) ; rôle des 
interfaces dans les systèmes composites; influence de la composition, des instabilités structurales et de la microstructure sur $T_{\mathrm{cr}}, H_{\mathrm{cr}}$ (champ critique) et $J_{\mathrm{cr}}$ (courant critique).

On montrera par ailleurs que les recherches dans le domaine des matériaux supraconducteurs qui peuvent être source d'idées nouvelles et de phénomènes nouveaux s'orientent dans les directions suivantes : (1) coexistence magnétisme-supraconductivité (2) recherche de la supraconductivité de type $p$ ou triplet (3) supraconductivité et onde de densité de charge dans les composés de basse dimensionalité (4) les supraconducteurs organiques.

\section{Rappels généraux sur les supraconducteurs}

Lors du refroidissement d'un supraconducteur audessous d'une certaine température $T_{\mathrm{cr}}$, appelée température critique, la tension nécessaire pour faire passer un courant continu donné s'annule, ce qui confère au matériau la capacité de conduire le courant sans dissipation d'énergie.

Le comportement des matériaux supraconducteurs en présence d'un champ magnétique extérieur permet de les classer en deux catégories.

\subsection{LES SUPRACONDUCTEURS DE PREMIÈRE ESPÈCE} OU DE TYPE I. - Lorsqu'un matériau, susceptible de devenir supraconducteur, est soumis à un champ magnétique $H$ et que l'on mesure l'induction magnétique $B(H)$ créée dans le matériau on constate que (a) pour $H<H_{\mathrm{c}}(T)$, l'induction est nulle dans le matériau, qui se comporte comme un diamagnétique parfait, c'est l'effet Meissner (b) pour $H>H_{\mathrm{c}}(T)$, l'induction magnétique pénètre complètement dans le matériau et ce dernier repasse brusquement à l'état normal. La supraconductivité est donc détruite au-delà d'une valeur du champ critique $H_{c}$ qui croît lorsque la température diminue.

2.2 LES SUPRACONDUCTEURS DE DEUXIÈME ESPÈCE OU DE TYPE II. - Reprenant la même expérience que précédemment, on peut constater le comportement suivant dans un très grand nombre de matériaux et notamment dans les composés binaires et ternaires (a) pour $H<H_{\mathrm{cl}}, B=0$ l'effet Meissner est observé (b) pour $H_{\mathrm{c} 1}(T)<H<H_{\mathrm{c} 2}(T)$ le conducteur est dans un état mixte, ce qui signifie que l'induction magnétique pénètre par tubes de flux quantifiés $\left(\Phi_{0}=\frac{h}{2 e}\right)$ dont le nombre va en augmentant avec le champ magnétique extérieur ; cet état disparaît au-delà de $H_{\mathrm{c} 2}$.

Deux champs critiques $H_{\mathrm{c} 1}$ et $H_{\mathrm{c} 2}$, qui augmentent lorsque la température décroît, caractérisent les supraconducteurs de type II.

Cette classification des matériaux supraconducteurs peut se retrouver en faisant appel à d'autres paramètres qui résultent des travaux théoriques de Ginzburg-Landau, Frölhlich en 1950 [1] qui ont conduit à l'élaboration de la théorie microscopique de la supraconductivité par Bardeen-CooperSchrieffer en 1957 [2]. Universellement reconnue, cette théorie repose sur la formation dans l'état supraconducteur de paires d'électrons de spins et de moments opposés dites paires de Cooper. L'interaction attractive électron-électron s'effectue par l'intermédiaire du réseau, donc des phonons. La comparaison de deux longueurs caractéristiques permet de distinguer les supraconducteurs de classe I et de classe II :

- la longueur de cohérence $\xi(T)$ : c'est la distance moyenne sur laquelle existent des corrélations entre électrons ; elle caractérise les modulations spatiales du paramètre d'ordre,

- la longueur de pénétration $\lambda(T)$ : dans l'expérience simple d'un demi-espace supraconducteur et d'un champ magnétique parallèle à la surface, l'induction magnétique $\mathrm{B}$ à l'intérieur du supraconducteur varie exponentiellement avec une constante de décroissance $\lambda$.

On définit alors le rapport de Ginzburg-Landau $K=\frac{\lambda(T)}{\xi(T)}$. Pour $K<\frac{\sqrt{2}}{2}$ les supraconducteurs sont de type I. Pour $K>\frac{\sqrt{2}}{2}$ les supraconducteurs sont de type II.

Le caractère supraconducteur d'un matériau disparaît au-delà de valeurs critiques de la température $T_{\text {cr }}$, du champ magnétique $H_{\mathrm{c}}$ (type I), $H_{\mathrm{c} 2}$ (type II) et de la densité de courant $J_{\mathrm{cr}}$, traversant le matériau. On définit alors dans une espace à trois dimensions $\left(T_{\mathrm{cr}}, H_{\mathrm{c}}\right.$ et $\left.J_{\mathrm{cr}}\right)$ une surface de transition pour chaque supraconducteur.

Quelles que soient les applications de la supraconductivité, il sera toujours tentant d'utiliser les matériaux qui présenteront la température critique la plus élevée. La chronologie des recherches montre que depuis plus de douze ans aucun supraconducteur n'a été découvert avec une température critique supérieure à $23,2 \mathrm{~K}$, celle correspondant à $\mathrm{Nb}_{3} \mathrm{Ge}[3,4]$. Selon la théorie classique les températures critiques sont d'autant plus élevées que l'interaction électronphonon et la densité d'états au niveau de Fermi $N\left(E_{F}\right)$ sont plus fortes. Ainsi à travers l'interaction électron-phonon semble se manifester l'aversion de la nature pour les réseaux cristallins très instables susceptibles d'engendrer de hautes températures critiques.

\section{Problèmes et perspectives d'utilisation des maté- riaux supraconducteurs}

Ce sont les températures, le champ et la densité de courant critiques qui détermineront le domaine d'application des supraconducteurs. On distinguera (a) les applications en courant fort : en continu, régime variable ou alternatif (électrotechnique, champs magnétiques intenses), (b) les applications en microélectronique (Tableau I).

\subsection{LES APPLICATIONS EN COURANT FORT.}

3.1.1 Problèmes métallurgiques. - Les applications en courant fort nécessitent des champs et densité de 
Tableau I. - Applications des matériaux supraconducteurs en courant fort et en microélectronique.

MATÉRIAUX SUPRACONDUCTEURS

\section{APPLICATIONS}

\section{EN COURANT FORT}

\section{EN RÉGIME A \\ VARIATIONS \\ LIMITÉES}

COURANT

CONTINU

- Chambre à bulles*

Contrôle de faisceaux de particules*

- Aimants MHD

- Inducteurs

- Inducteur homolaire

- Tri magnétique

- Inducteurs de machines synchrones

- Tore pour fusion thermonucléaire*

- Limiteur de courant

- Câbles

- Remnographie médicale* - Stockage magné-
tique*

\section{EN COURANT} ALTERNATIF

- Générateurs synchrones

- Transformateurs

- Prédisjoncteurs

- Limiteur de courant

- Régulateur de tension
MICROÉLECTRONIQUE
- Squid*

- Transistor (QUITERON)

- Détecteur optique

- Lignes de transmission et composants passifs

- Echantillonneur Josephson

\section{$\mathrm{NbTi}$}

$\mathrm{Nb}_{3} \mathrm{Sn}$

$\mathrm{V}_{3} \mathrm{Ga}$
MATÉRIAUX UTILISÉS

$\mathrm{Pb}, \mathrm{Nb}, \mathrm{NbN}$

\section{MARCHÉ EXISTANT*}

courant critiques élevés, ce sont donc les supraconducteurs de type II qui seront utilisés. Du fait de leur grande ductilité, les alliages $\mathrm{Nb}-\mathrm{Ti}$ de structure c.f.c., sous forme de monofilament ou de multifilaments, sont les plus commercialisés. Cependant d'autres matériaux tels que $\mathrm{Nb}_{3} \mathrm{Sn}$ et $\mathrm{V}_{3} \mathrm{Ga}$ ayant une structure isotype de celle du tungstène $\beta$, appelés encore composés A-15, connaissent actuellement un développement industriel sous forme de multifilaments et de rubans. Bien que découverts il y a plus de trente ans, les composés A-15 ont vu leur industrialisation retardée du fait de difficultés métallurgiques pour leur mise en forme ; mécaniquement fragiles ils sont peu ductiles.

Pour comprendre les étapes des procédés métallurgiques utilisés et les difficultés rencontrées pour la fabrication d'aimants supraconducteurs, il suffit de se rappeler que les supraconducteurs de type II dans l'état mixte sont pénétrés partiellement par l'induction magnétique sous forme d'un réseau de canaux entourés des supracourants appelés vortex. L'ensem- ble ligne de flux-vortex, appelé fluxoïde, soumis à un courant se déplace sous l'action des forces de Lorentz, ce qui peut engendrer un échauffement local et provoquer le retour du supraconducteur à l'état normal.

Lorsque le supraconducteur est traversé par un courant alternatif la situation est beaucoup plus défavorable en raison des pertes hystérétiques qui ne sont autres que celles engendrées par le déplacement des vortex, imposé par la nature variable du champ magnétique et la variation induite de $B$ dans le matériau. Par ailleurs des causes diverses telles que des actions mécaniques, électriques ou des champs pulsés produits par des impulsions de courant peuvent conduire à des déplacements des fluxoïdes. Pour pallier ces inconvénients plusieurs solutions sont utilisées :

a) Les fils ou rubans d'un aimant supraconducteur par exemple sont noyés dans une gaine de cuivre qui accroît la résistance mécanique des bobines et joue le rôle de stabilisant cryostatique. Lorsqu'il y a 
transition de l'état supraconducteur à l'état normal, la chaleur qui en résulte est dérivée dans le cuivre dont la conductivité est plus grande que celle des supraconducteurs à l'état normal.

b) Pour limiter les pertes hystérétiques en régime variable ou alternatif, on est conduit à réduire le diamètre du supraconducteur puisque ces pertes sont proportionnelles à son diamètre. Les pertes hystérétiques, par unité de volume de supraconducteur, sont données en première approximation par la relation

$$
P=\frac{2}{3 \pi} d J_{\mathrm{cr}}\left[1+\left(\frac{J}{J_{\mathrm{cr}}}\right)^{2}\right] \frac{\mathrm{d} B}{\mathrm{~d} t}
$$

dans laquelle $J_{\mathrm{cr}}$ est la densité de courant critique du supraconducteur, $J$ est la densité du courant transporté par les filaments et $d$ le diamètre de ces derniers. On voit que cette relation conduit à rechercher des filaments de diamètre le plus faible possible [5]. Cependant la double préoccupation de transport de courant élevé (donc section importante du supraconducteur) et la difficulté d'utilisation de monofilaments de faible diamètre a conduit à une structure multifilamentaire du supraconducteur. Avec une telle configuration de type multifilamentaire surgit une autre difficulté, celle de la présence de courants de Foucault au sein de la matrice de cuivre, qui se dissipent en chaleur. Ce problème a été résolu d'une part par un enrobage plus résistif des filaments (utilisation du cupro-nickel) et d'autre part par un torsadage aussi serré que possible des filaments. En effet, les pertes par courants induits dépendent du pas de torsion des filaments $\lambda$ et de la résistivité transversale équivalente de la matrice $\rho_{\mathrm{M}}$. Par unité de volume du brin on a :

$$
P=\left(\frac{\lambda}{2 \pi} \frac{\mathrm{d} B}{\mathrm{~d} t}\right)^{2} \frac{K}{\rho_{\mathrm{M}}}
$$

où $K$ est un facteur sans dimension dépendant de la structure multifilamentaire [5].

c) Enfin, mis à part les problèmes de dissipation d'énergie l'attention sera focalisée sur la capacité de transport du courant dans les filaments. Il faudra donc rechercher, pour obtenir des champs magnétiques intenses, des densités de courant critique aussi élevées que possible.

L'aptitude des filaments supraconducteurs à transporter un courant de forte intensité sera conditionnée par leur capacité à piéger les fluxoïdes. Ce piégeage, qui dépend très fortement des imperfections chimiques et structurales des filaments supraconducteurs donc de leur microstructure, influe directement sur la valeur des densités de courant critique.

La recherche de la microstructure la plus favorable sera le fait d'une combinaison judicieuse de traitements thermiques et mécaniques des filaments. $\mathrm{Ce}$ sera donc en allant vers une approche empirique du problème que se dégagera la meilleure solution [5-7].
Toutes ces généralités peuvent être illustrées par quelques exemples. Ainsi dans les alliages $\mathrm{Nb}-\mathrm{Ti}$ actuellement les plus utilisés industriellement, c'est la présence de précipités d'une phase riche en titane qui, pour un espacement et une taille bien contrôlés, assurent le meilleur ancrage possible des fluxoïdes et par conséquent permettent l'obtention de courants critiques élevés $[5,7,8]$. Même dans le composé $\mathrm{Nb}_{3} \mathrm{Sn}$ il a été démontré que la densité de courant critique est d'autant plus grande que les joints de grains, qui sont des pièges pour les fluxoïdes, sont plus nombreux et par conséquent que la taille des grains est plus faible [9]. Il semblerait en être de même pour $\mathrm{Nb}_{3} \mathrm{Ge}$ [10].

Notons que la présence de joints de grains n'est pas seulement responsable de l'ancrage des fluxö̈des, l'introduction d'impuretés ou la présence de phases parasites peuvent jouer le même rôle. Ainsi avec $1.000 \mathrm{ppm}$ d'azote et $1.000 \mathrm{ppm}$ d'oxygène, la densité de courant critique est presque doublée dans les alliages $\mathrm{Nb}-\mathrm{Ti}$ [5]. Demolliens a montré récemment que pour $\mathrm{Nb}_{3} \mathrm{Ge}$ préparé sous forme de ruban, le courant critique le plus élevé est observé dans les échantillons contenant environ $10 \%$ en masse de la phase parasite $\mathrm{Nb}_{3} \mathrm{Ge}_{5}$ [10].

Les impuretés présentes dans les matériaux supraconducteurs peuvent jouer un rôle autre que celui de piéger les fluxoïdes. Elles peuvent en diminuant le libre parcours moyen des électrons $l$ et par conséquent la longueur de cohérence $\xi$, contribuer à augmenter la densité de courant critique en élevant le champ critique. Pour cela il suffit de se rappeler d'une part que le champ critique est relié à la longueur de cohérence par la relation

$$
\mu_{0} H_{\mathrm{c} 2}=\frac{\phi_{0}}{2 \pi \xi^{2}}\left(\phi_{0}=\text { quantum de flux }\right)
$$

et d'autre part que $\xi \alpha\left(\frac{V_{\mathrm{F}}}{T_{\mathrm{cr}}} l\right)^{1 / 2}\left(V_{\mathrm{F}}=\right.$ vitesse des électrons au niveau de Fermi). La raison physique de la relation entre $H_{\mathrm{c} 2}$ et $\xi$ est que la dimension du cœur du fluxoïde est de l'ordre $\xi$ et que par conséquent plus $\xi$ sera petit plus le matériau acceptera de lignes de flux et aura donc un champ critique élevé. Le fait que la longueur de cohérence soit petite implique un faible parcours moyen pour les électrons et une résistivité élevée pour le matériau. Ainsi une augmentation de la résistivité de $\mathrm{Nb}_{3} \mathrm{Sn}$ par dopage avec des impuretés métalliques (Ti, Ta ou $\mathrm{Ni}+\mathrm{Zn}$ ) accroît le champ critique et la densité de courant critique sans toutefois affecter notablement la température critique [9].

Il est important de noter que en plus des problèmes de microstructure qui ont été évoqués, ceux concernant la diffusion aux interfaces entre les filaments et la matrice d'enrobage ont une influence importante sur les densités de courant critique et les propriétés mécaniques des structures multifilamentaires. La précipitation de composés non ductiles comme $\mathrm{Ti}_{2} \mathrm{Cu}$ à l'interface filament $\mathrm{Nb}-\mathrm{Ti} / /$ matrice- 
$\mathrm{Cu}$ [7] ou bien la contamination de la matrice de cuivre par l'étain dans la fabrication des filaments de $\mathrm{Nb}_{3} \mathrm{Sn}$ par la technique du «bronze » peuvent être éliminées par l'insertion de barrières antidiffusion.

3.1.2 Perspectives d'utilisation. - En général pour des applications nécessitant des champs magnétiques allant jusqu'à 9-10 T, l'alliage Nb-Ti est utilisé. Pour l'obtention de champs supérieurs compris entre 9 et $14 \mathrm{~T}, \mathrm{Nb}_{3} \mathrm{Sn}$ sera choisi et pour des champs plus élevés mais néanmoins inférieurs à $30 \mathrm{~T}$, on peut faire appel à $\mathrm{V}_{3} \mathrm{Ga}$. Les travaux de Demolliens sur la production de rubans de $\mathrm{Nb}_{3} \mathrm{Ge}$ par transport chimique en phase vapeur, semblent prometteurs puisque des courants critiques de $1,6 \times 10^{4} \mathrm{~A} / \mathrm{cm}^{2}$ à $4,2 \mathrm{~K}$ et $2,1 \times 10^{4} \mathrm{~A} / \mathrm{cm}^{2}$ à $1,8 \mathrm{~K}$ sous $20 \mathrm{~T}$ ont été obtenus [10].

Notons par ailleurs les efforts réalisés par Alsthom-Atlantique et les laboratoires de la Compagnie Générale d'Electricité pour développer des multifilaments de hautes performances en alliage $\mathrm{Nb}-\mathrm{Ti}$ : des brins de 15000 filaments de $0,6 \mu \mathrm{m}$ $\left(J_{\mathrm{cr}} \sim 2 \times 10^{5} / \mathrm{cm}^{2}-5 \mathrm{~T}-4,2 \mathrm{~K}\right)$ pouvant supporter des impulsions de $1000 \mathrm{~T} / \mathrm{s}$ sont disponibles; des brins de 250000 filaments de $0,08 \mu \mathrm{m}$ et $0,02 \mu \mathrm{m}$ sont en cours de développement [11-13].

L'année 1983 peut être considérée à plusieurs titres comme une étape notable du développement industriel des applications de la supraconductivité (régulateur de puissance à stockage magnétique, accélérateurs de particules, remnographes médicaux, cryoalternateurs, bobines supraconductrices à fréquences industrielles...). On voit donc brusquement apparaître les applications de la supraconductivité dans de nombreux domaines après 20 années de recherche et développement. La tendance sera de poursuivre les efforts vers des matériaux ayant des champs critiques de plus en plus élevés. Ces matériaux existent, ce sont les phases dites de Chevrel découvertes à Rennes en 1971 [14]. Avec une température critique de $15 \mathrm{~K}$ environ pour $\mathrm{PbMo}_{6} \mathrm{~S}_{8}$, le champ critique avoisinant $50-60 \mathrm{~T}$ à $4,2 \mathrm{~K}$, est environ deux fois plus élevé que celui de $\mathrm{Nb}_{3} \mathrm{Sn}$ et plus de quatre fois plus grand que celui des alliages $\mathrm{Nb}-\mathrm{Ti}$. Sergent, Chevrel et al. ont réalisé en collaboration avec divers laboratoires universitaires et industriels (CGE, Alsthom, CEA, CENG) un brin de 6 filaments de $65-120 \mu \mathrm{m}$ et d'une longueur de $300 \mathrm{~m}$ [15]. La densité de courant critique $\left(J_{\mathrm{cr}}=\right.$ $10^{7}-10^{8} \mathrm{~A} / \mathrm{m}^{2}-5 \mathrm{~T}-4,2 \mathrm{~K} ; J_{\mathrm{cr}}=1,5-2,5 \times 10^{7} \mathrm{~A} / \mathrm{cm}^{2}$ $9 \mathrm{~T}-4,2 \mathrm{~K}$ ) demeure encore relativement faible, mais les résultats obtenus sont comparables à ceux publiés par les chercheurs suisses, américains ou japonais [16-18].

Enfin, signalons que des composés appartenant à la famille des phases de Laves, $\mathrm{V}_{2}$ ( $\mathrm{Hf}, \mathrm{Zr}$ ) élaborés sous forme multifilamentaire, pourraient concurrencer largement des composés A-15 $\left(H_{\mathrm{c} 2}>20 \mathrm{~T}\right.$ à 4,2 K) surtout pour l'insensibilité de leurs propriétés supraconductrices aux contraintes mécaniques et à l'irradiation par les neutrons [19].
3.2 APPLICATIONS EN MICROÉLECTRONIQUE. Les matériaux supraconducteurs envisagés actuellement pour les applications en microélectronique doivent satisfaire aux critères qu'imposent la physique des barrières tunnels et la technologie des jonctions.

Les orientations de recherche sont les suivantes :

- Pour les électrodes supraconductrices.

Utilisation de matériaux à hautes $T_{\mathrm{cr}}$. Le but est de réaliser des jonctions dont la densité de courant varie peu avec les fluctuations de température et de pouvoir utiliser des microréfrigérateurs fonctionnant autour de $10 \mathrm{~K}$. Les efforts portent sur $\mathrm{Nb}, \mathrm{NbN}$ et quelques composés de type $\mathrm{A}_{15}\left(\mathrm{~V}_{3} \mathrm{Si}, \mathrm{Nb}_{3} \mathrm{Sn}\right.$, $\left.\mathrm{Nb}_{3} \mathrm{Ge} . ..\right)[20,21]$.

\section{- Pour les barrières tunnels.}

Utilisation de matériaux de faible constante diélectrique et par conséquent de faible capacité. Les études concernent les barrières d'oxydes natifs résultant de l'oxydation de l'électrode supraconductrice elle-même et les barrières tunnels artificielles semiconductrices (le silicium amorphe ou le germanium) qui sont utilisées lorsque l'électrode supraconductrice ne forme pas un oxyde natif de bonne qualité comme dans le cas des composés $\mathrm{A}-15, \mathrm{Nb}_{3} \mathrm{Ge}$ par exemple. Les barrières semi-conductrices en silicium amorphe ont une faible constante diélectrique et le fait qu'elles aient une épaisseur plus grande, conduit à des jonctions de faible capacité [22].

Notons que se développent également les barrières multicouches oxydées qui mettent en jeu les effets de proximité responsables de la supraconductivité d'une couche très mince de métal normal déposée sur un film supraconducteur [22].

C'est dans les applications en microélectronique que les supraconducteurs de type I trouvent une de leurs rares applications, le plomb par exemple comme électrode supraconductrice dans les jonctions Josephson ou l'indium pour des détecteurs de neutrinos [23].

\section{Nouvelles orientations des recherches}

Les nouveautés qui apparaissent actuellement en supraconductivité ne résultent pas uniquement de l'étude du comportement de supraconducteurs bien connus sous l'action de perturbations extérieures qui ont permis par exemple la découverte du Quiteron (combinaison de deux jonctions tunnels supraconductrices où des effets hors d'équilibre conduisent à l'apparition d'un fonctionnement de type transistor).

Grâce à la recherche systématique de nouveaux composés supraconducteurs les études se développent actuellement dans plusieurs directions pour montrer par exemple que le ferromagnétisme et la supraconductivité ne sont pas des phénomènes qui s'excluent mutuellement et qu'il peut exister un type de supraconductivité (état triplet) autre que celui qui est à la base de la théorie microscopique de BCS (état singlet). Par ailleurs l'étude des conditions d'existence de la supraconductivité dans des matériaux de basse dimensionalité (compatibilité avec les 
ondes de densité de charge) et dans les composés organiques fait l'objet de nombreux travaux.

4.1 COEXISTENCE MAGNÉTISME-SUPRACONDUCTIVITÉ. - La coexistence du magnétisme et de la supraconductivité met en confrontation deux phénomènes collectifs, ce qui donne naissance à de nouvelles propriétés. Les travaux théoriques de Ginzburg en 1957 [24] et de Abrikosov et Gor'kov en 1961 [25] montrent que les impuretés magnétiques en interagissant fortement avec les électrons itinérants détruisent les paires de Cooper et par conséquent la supraconductivité. Les travaux expérimentaux de Matthias et al. [26] en 1958 confirment les résultats théoriques.

Il est utile de rappeler que les paires de Cooper peuvent être détruites par deux effets: (a) une diffusion par les impuretés magnétiques (effet du second ordre), (b) la présence d'un champ d'échange (effet de premier ordre) résultant de l'existence d'un champ magnétique extérieur ou bien de l'établissement d'un ordre magnétique.

L'hamiltonien de l'interaction d'échange entre le spin de l'impureté magnétique $S$ et celui des électrons de conduction $s$ est donné par l'expression: $\mathfrak{H}_{\mathrm{cx}}=-j$ Șs où $j$ est le paramètre de l'interaction d'échange.

Quel que soit l'ordre magnétique, antiferromagnétique ou ferromagnétique qui entrera en compétition avec la supraconductivité, il sera indispensable que $j$ soit faible pour observer la coexistence des deux phénomènes.

4.1.1 Coexistence supraconductivité-antiferromagnétisme. - La coexistence supraconductivité-antiferromagnétisme a été observée pour la première fois dans les phases de Chevrel $\mathrm{REMo}_{6} \mathrm{~S}_{8}(\mathrm{RE}=\mathrm{Gd}$, $\mathrm{Tb}, \mathrm{Dy}, \mathrm{Er}), \mathrm{REMo}_{6} \mathrm{Se}_{8}(\mathrm{RE}=\mathrm{Gd}, \mathrm{Tb}, \mathrm{Er})$ et les borures ternaires $\mathrm{RERh}_{4} \mathrm{~B}_{4} \quad(\mathrm{RE}=\mathrm{Nd}, \quad \mathrm{Sm}$, $\mathrm{Tm})$ [27]. Les électrons itinérants $4 \mathrm{~d}$ qui résultent de la présence d'amas de métaux de transition $\mathrm{Mo}_{6}$ pour les phases de Chevrel et $\mathrm{Rh}_{4}$ pour les borures, sont faiblement couplés aux ions magnétiques de terres rares; $|j| \sim 0,01 \mathrm{eV}$ pour les phases de Chevrel [27].

4.1.2 Coexistence supraconductivité-ferromagnétis$m e$. - Sans préjuger de la nature microscopique de la supraconductivité, Ginzburg [24] prévoyait dès 1957 la très grande difficulté qu'il y aurait à découvrir des matériaux où coexisteraient le ferromagnétisme et la supraconductivité. L'induction créée par l'aimantation spontanée peut atteindre $20 \mathrm{kG}$ et ainsi détruire la supraconductivité de tout composé à faible champ critique. Il prédisait que ferromagnétisme et supraconductivité ne pourraient coexister que : (a) dans des composés ayant un champ critique plus grand que l'induction spontanée, (b) dans les régions d'un échantillon où l'induction magnétique est considérablement réduite.

Pour démontrer les idées de Ginzburg il paraissait naturel d'étudier des matériaux dans lesquels le ferromagnétisme et la supraconductivité avaient été observés, soit des composés à supraconductivité réentrante qui s'ordonnent ferromagnétiquement à température plus basse que celle où apparaît la transition supraconductrice.

Ce serait donc dans les parois de Bloch des domaines ferromagnétiques, aimantés alternativement dans un sens et dans l'autre que pourrait apparaitre la supraconductivité.

Trois composés à supraconductivité réentrante ont été étudiés [28] : $\mathrm{ErRh}_{4} \mathrm{~B}_{4}$ de structure quadratique type $\mathrm{CeCo}_{4} \mathrm{~B}_{4}$ caractérisé par un plan de facile aimantation, $\mathrm{HoMo}_{6} \mathrm{~S}_{8}$ de symétrie rhomboédrique présentant un axe de facile aimantation [111] et $\mathrm{Sn}_{1-x}^{(1)} \operatorname{Er} \underset{(x)}{(1)} \mathrm{Er}_{4}^{(2)} \mathrm{Rh}_{6} \mathrm{Sn}_{18}$ de structure quadratique.

Des mesures de résistivité électrique ou de susceptibilité alternative ont été réalisées sur des monocristaux des composés signalés précédemment. Une résistance nulle, caractérisant un état supraconducteur a été observée lorsque les contacts électriques sont disposés parallèlement aux parois de Bloch soit dans la direction de l'axe de facile aimantation pour $\mathrm{HoMo}_{6} \mathrm{~S}_{8}$ et dans le plan de facile aimantation pour $\mathrm{ErRh}_{4} \mathrm{~B}_{4}$. Une résistance pratiquement égale à celle de l'état normal a été observée perpendiculairement aux parois de Bloch.

4.1.3 Supraconductivité induite par un champ magnétique extérieur. - Aussi surprenant que cela puisse paraître à première vue, la supraconductivité peut être induite par un champ magnétique extérieur dans certains composés de terres rares.

4.1.3.1 Supraconductivité induite en champ intense dans le composé paramagnétique $\mathrm{Eu}_{x} \mathrm{Sn}_{1-x}$ $\mathrm{Mo}_{6} S_{8}$ [29]. - Pour $x=0,8$ et $T<1 \mathrm{~K}$ la supraconductivité disparaît pour un champ très faible (0,6-1 T) pour réapparaitre à plus haut champ vers 8 tesla et disparaître à nouveau au-delà de 20 tesla. L'apparition de la supraconductivité entre 8 et 20 tesla résulte tout simplement du fait que le champ d'échange interne $H_{\text {ech }}$ et le champ extérieur $H_{\text {ext }}$ sont opposés parce que $j<0$. Les électrons de conduction ne voient alors que la différence $H_{\text {ech }}-H_{\text {ext }}$ et par conséquent ne peuvent s'associer en paires de Cooper que pour une certaine valeur faible du champ magnétique résultant, c'est l'effet Jaccarino-Peter. Ce type de phénomène n'a pu être observé que dans des composés à champ critique élevé et donc caractérisés par une faible longueur de cohérence $\xi$.

4.1.3.2 Supraconductivité induite en champ faible dans un composé ferromagnétique. - Des mesures de résistivité sur des monocristaux de $\mathrm{HoMo}_{6} \mathrm{~S}_{8}$ ont montré que la supraconductivité apparaissait lorsque le champ magnétique extérieur appliqué $H_{\text {ext }}$ était opposé à l'aimantation rémanente $\left(H_{\mathrm{ext}} \sim 0,1 \mathrm{~T}\right.$ à $25 \mathrm{mK})$ [28].

4.2 A LA RECHERCHE DE LA SUPRACONDUCTIVITÉ TRIPLET OU DE TYPE p. - La découverte de l'hélium 3 superfluide a conduit à rechercher un nouveau type de supraconductivité dans les solides 
autres que celui proposé par BCS (état singlet). Supraconductivité et superfluidité sont deux propriétés jumelles de la nature. La première concerne les métaux, la seconde les gaz ou liquides neutres. Les atomes $\mathrm{d}^{3} \mathrm{He}$, pour devenir superfluides, ont besoin de s'associer par paires comme les électrons des supraconducteurs. On sait que dans l'un de leurs états superfluides, les paires $d^{33} \mathrm{He}$ sont anisotropes (spins nucléaires parallèles) [30]. Ceci a conduit à se demander, par analogie avec ${ }^{3} \mathrm{He}$ superfluide, pourquoi il n'existerait pas un nouveau type de supraconductivité qui serait caractérisé pas des paires d'électrons à spins parallèles (état triplet ou de type $p$ ).

Les recherches se sont naturellement orientées vers des composés dont le comportement de la chaleur spécifique à très basse température présentait des analogies avec celui de l'hélium 3 : variation non exponentielle de la chaleur spécifique en dessous de $T_{\mathrm{cr}}$, variation en lois de puissance (en $T^{2}$ par exemple) de la conductivité thermique, de l'atténuation des ultrasons, du pouvoir thermoélectrique...

Par ailleurs des travaux théoriques montrent qu'une supraconductivité de type $\mathrm{p}$ peut être induite dans des composés proches du magnétisme présentant des fluctuations de spins (paramagnons) « presque » ferromagnétiques ou « presque » antiferromagnétiques. La présence de paramagnons est favorable à l'apparition de la supraconductivité triplet et défavorable à l'existence de la supraconductivité de type BCS (état singlet) [31]. Notons également que l'appariement de type $\mathrm{p}$ des électrons est facilement contrarié par la présence d'impuretés magnétiques ou non magnétiques $[32,33]$.

Toutes ces propriétés semblent se manifester dans des composés présentant un très fort terme $\gamma$ de chaleur spécifique électronique que l'on appelle actuellement composés à " fermions lourds ». Ce sont soit des composés binaires ou ternaires contenant du cérium ou de l'uranium comme par exemple : $\mathrm{CeAl}_{3}, \mathrm{CeCu}_{6}, \mathrm{UPt}_{3}, \mathrm{UBe}_{13}, \mathrm{CeCu}_{2} \mathrm{Si}_{2}$. Parmi les exemples cités seuls les trois derniers composés deviennent supraconducteurs respectivement à $0,54 \mathrm{~K}, 0,9 \mathrm{~K}$ et $0,5 \mathrm{~K}$ [34]. Le type de supraconductivité auquel appartient chacun de ces composés n'est pas encore bien défini. La variation thermique de leur chaleur spécifique à basse température n'est pas exponentielle en dessous de $T_{\mathrm{cr}}$; des lois de puissance notamment en $T^{2}$ sont observées pour un certain nombre de propriétés de $\mathrm{UPt}_{3}$ : conductivité thermique, chaleur spécifique, pouvoir thermoélectrique et atténuation des ultrasons [35]. Pour $\mathrm{UBe}_{13}$ bien en dessous de $T_{\mathrm{cr}}$, la chaleur spécifique varie en $T^{3}$ et la conductivité thermique en $T^{2}[34,35]$. Toutes ces propriétés semblent indiquer que la supraconductivité pourrait être de type $\mathrm{p}$ dans $\mathrm{UBe}_{13}$ et $\mathrm{UPt}_{3}$. De plus pour $\mathrm{UPt}_{3}$, au-dessus de $T_{\text {cr }}$, la présence d'un terme $T^{3} \ln T$ dans la chaleur spécifique, qui est une manifestation de fluctuation de spins, renforce l'idée d'une supraconductivité de type $p[34,35]$. La situation est nettement moins claire pour $\mathrm{CeCu}_{2} \mathrm{Si}_{2}$ pour lequel des mesures récen- tes de courant tunnel en fonction d'un champ magnétique extérieur seraient plutôt en faveur d'une supraconductivité de type s (état singlet).

Bien qu'il y ait de fortes présomptions d'observer une supraconductivité de type $\mathrm{p}$ dans les composés à fermions lourds, il n'existe pas encore de preuves vraiment décisives. En fait la démonstration claire de l'existence d'une supraconductivité de type $p$ ne pourra se faire que grâce à la découverte de matériaux supraconducteurs ferromagnétiques itinérants dans lesquels les mêmes électrons seront responsables à la fois du magnétisme et de la supraconductivité. Il semblerait que le composé $\operatorname{ErRh}_{4} B_{3,8} \square_{0,2}$, de symétrie quadratique et de structure type $\mathrm{LuRu}_{4} \mathrm{~B}_{4}$, possède cette propriété [37].

\subsection{SUPRACONDUCTIVITÉ ET MATÉRIAUX DE BASSE} DIMENSIONNALITÉ. - De nombreux composés à dimensionnalité restreinte tant bidimensionnels qu'unidimensionnels présentent des transitions structurales à basse température dites transitions de Peierls. Ces instabilités structurales ne se produisent que dans la mesure où une déformation du cristal est nécessaire pour bénéficier d'un gain d'énergie électronique via une modulation de la densité électronique appelée onde de densité de charge (ODC).

L'étude de $\mathrm{NbSe}_{2}$ (bidimensionnel) et $\mathrm{NbSe}_{3}$ (unidimensionnel) qui présentent une ODC et des propriétés supraconductrices a permis de montrer le lien entre ces deux phénomènes qui, tous les deux, font intervenir l'interaction électron-phonon.

Soumis à une pression, $\mathrm{NbSe}_{2}$ voit sa température critique s'élever alors que l'ODC est progressivement supprimée [38]. $\mathrm{NbSe}_{3}$ unidimensionnel, présente deux ODC ; il n'est pas supraconducteur à la pression normale au-dessus de $7 \mathrm{mK}$. Il devient supraconducteur sous-pression lorsque disparaît l'ODC [39]. L'ODC est donc un poison de la supraconductivité.

\section{Conclusions}

Malgré les difficultés de la théorie microscopique de la supraconductivité de faire des prédictions de température critique, elle fait néanmoins clairement apparaître les principaux paramètres qui gouvernent le phénomène : densité d'état au niveau de Fermi, spectre de phonons et interaction électron-phonon. Le chercheur qui observe les caractéristiques physiques des supraconducteurs en vue de les améliorer est conduit, en dehors de la nécessité de préparer des matériaux purs, à agir sur le type de réseau cristallin et la stoechiométrie. Il est plus aisé d'agir dans le sens de la dernière proposition que de suivre la première dans la mesure où les aspects structuraux prévisionnels restent encore peu maîtrisables. Disposant donc d'un modèle structural, il sera alors assez facile de modifier d'une manière contrôlée le nombre de porteurs et la densité d'états en jouant essentiellement sur la composition, les effets de désordre ou de substitution. Les composés en type A-15 illustrent très bien cette approche. 
Pour progresser dans la recherche de nouveaux matériaux supraconducteurs, il ne faut pas s'enfermer dans des idées préconçues qui seraient le fruit de modèles empiriques trop simplistes. Il faut au contraire envisager des études systématiques sur de nouvelles familles de composés. La plupart des composés supraconducteurs intéressants n'ont-ils pas été découverts fortuitement ? Sans préjuger des recherches futures, on peut toutefois penser qu'avec le très grand potentiel permettant de former de nouveaux composés avec trois éléments différents, les nouveaux supraconducteurs seront encore pour longtemps des matériaux ternaires.

Dans le domaine des applications immédiates, l'intérêt se situe sur un autre plan. Il est clairement apparu que de meilleurs contrôle et caractérisation de la microstructure des matériaux connus sont primordiaux. Ceci est vrai à la fois pour $\mathrm{NbTi}$ et $\mathrm{Nb}_{3} \mathrm{Sn}$, déjà commercialisés ; de même pour les matériaux de la prochaine génération, comme $\mathrm{Nb}_{3} \mathrm{Ge}$ et $\mathrm{PbMo}_{6} \mathrm{~S}_{8}$. Notons que pour les applications en courant alternatif qui nécessitent des filaments de faible diamètre, c'est l'alliage $\mathrm{Nb}$-Ti qui sera encore utilisé pour longtemps.

Il faut souligner l'impact que sont susceptibles d'avoir dans l'avenir les matériaux supraconducteurs en couches minces (a) pour élaborer de nouveaux matériaux métastables dont l'obtention aurait été impossible par des techniques conventionnelles, (b) pour déposer en rubans des matériaux pour lesquels l'extrusion est difficile.

En ce qui concerne les orientations nouvelles des recherches que ce soit pour étudier la coexistence supraconductivité-magnétisme ou la recherche de composés présentant une supraconductivité triplet ou de type $p$, les chimistes devront s'efforcer de préparer des monocristaux. Par ailleurs puisque la supraconductivité de type $\mathrm{p}$ semble apparaître dans des composés à forte chaleur spécifique électronique et que de plus elle est défavorisée par la présence d'impuretés magnétiques ou non magnétiques, les recherches devront se diriger vers des composés où le libre parcours moyen des électrons est nettement supérieur à la longueur de cohérence $(l \gg \xi)$.

Enfin on ne peut passer sous silence les supraconducteurs organiques dont l'intérêt essentiel se situe pour l'instant sur le plan de la recherche fondamentale. Notons que la température critique la plus élevée actuellement connue a été observée dans $\beta$ - (BEDT-TTF $)_{2} \mathrm{I}_{3}\left(T_{\text {cr }}=8,1 \mathrm{~K}\right)$. Ce résultat a été obtenu après l'application d'une pression hydrostatique d'hélium gazeux de 1,5 kbar et le relâchement de la pression à basse température [40].

\section{Bibliographie}

[1] FrÖhlich, H., Phys, Rev. 79 (1950) 845.

[2] BARDEen, J., CoOPER, A. S. and SChriefFer, J. R., Phys. Rev. 108 (1957) 1175.

[3] Gavaler, J. R., Appl. Phys. Lett. 23 (1973) 480.

[4] TESTARDI, L. R., WERNICK, J. H. and ROYER, W. A., Solid State Commun 15 (1974) 1.

[5] Maldy, J.,C Dubots, P. et Gérard, J., Rev. Gen. Elect. 88 (1979) 120.

[6] West, A. W. and Larbalestier, D. C., Metal Trans. A 15A (1984) 843.

[7] LARbalestier, D. C., IEE Trans. Magn. 21 (1985) 257.

[8] Larbalestier, D. C. and West, A. W., Ann. Chim. Fr. 9 (1984) 813.

[9] Suenaga, M., IEE Trans. Magn. 21 (1985) 1122.

[10] DemOlliens, O., Thèse Grenoble-INPG (1985).

[11] SABrIE, J. L., Communication privée et Ann. Chim. Fr. 9 (1984) 861.

[12] Hoang gia Ky, Gérard, J. et Dubots, P., Ann. Chim. Fr. 9 (1984) 823.

[13] Dubois, P., Dubots, P., FÉvrier, A., Renard, J. C., GoYer, J. and HoANG GIA Ky, Ann. Chim. 9 (1984) 833.

[14] Chevrel, R., Sergent, M. et Prigent, J., J. Solid State Commun. 3 (1971) 515.

[15] SERGENT, M., Vie des Sciences (à paraître), communication privée.

[16] Cattani, D., Baillif, R., Seeber, B., Fischer, O. and Glatzle, W., Proc. 9th Int. Conf. on Magnet Techn., Sept. 1985, Zürich (à paraître).

[17] EKIN, J. W., YAMAShITA, T. and HAMASAKI, K., IEE Trans. Magn. 21 (1985) 474.
[18] Hamasaki, K., Hirata, K., Yamashita, T. and KoMATA, T., IEE Trans. Magn. 21 (1985) 471.

[19] Inoue, K., Kuroda, T. and TachiKawa, K., IEE Trans. Magn. 21 (1985) 467.

[20] Villegier, J. C., VieuX-Rochaz, L., Goniche, M., RENARD, P. and VABRE, M., IEE Trans. Magn. 21 (1985) 498.

[21] TAlvaCChIO, J., BRAGinSKI, A. I., JANOCKO, M. A., IEE Trans. Magn. 21 (1985) 521.

[22] Adde, R. et Crozat, P., Ann. Chim. Fr. 9 (1984) 911.

[23] Waysand, G., Ann. Chim. Fr. 9 (1984) 805.

[24] Ginzburg, V. L., Sov. Phys. JETP 4 (1957) 153.

[25] Abrikosov, A. A. and Gorikov, L. P., Sov. Phys. JETP 12 (1961) 1243.

[26] Matthias, B. T., SUhl, H. and CoRenzwit, E., $J$. Phys. Chem. Solids 19 (1959) 346.

[27] MAPPle, M. B., in Advances in Superconductivity Ed. Dearer, B. and Ruvalds, J. (Plenum Press N.Y. and London) 1982, p. 279.

[28] Genicon, J. L., Tournier, R. et Modondanon, J. P., Courrier du CNRS, $\mathbf{N}^{\circ}$ Spécial 1986, Image des Matériaux (à paraître).

[29] Fischer, O., Ann. Chim. Fr. 9 (1984) 867.

[30] COMBESCOT, R., La Recherche 95 (1978) 1064.

[31] Anderson, P. W. and Brinkman, W. F., Phys. Rev. Lett. 30 (1973) 1108.

[32] Anderson, P. W. and Morel, P., Phys. Rev. 123 (1961) 1911.

[33] Balian, R. and Werthamer, N. R., Phys. Rev. 131 (1963) 1553

[34] Stewart, G. R., Rev. Mod. Phys. 56 (1984) 755.

[35] Sulpice, A., Gandit, P., Chaussy, J., FlouQuet, 
J., Jaccard, D., Lejay, P. and Tholence, J. L., J. Low Temp. Phys. 62 (1986) 39.

[36] OTt, H. R., RudigieR, H., Rice, T. M., UEDA, K., Fisk, Z., SMITH, J. L., Phys. Rev. Lett. 52 (1984) 1915.

[37] Chaussy, J., Genicon, J. L., Giroux, M., Odin, J., Shao, L. Q., Sulpice, A., Tournier, R., Chevalier, R., Lejay, P. et Etourneau, J., communication privée.

[38] Berthier, C., Jerome, D., Molinie, P. and RouXEL, J., Solid State Commun. 19 (1976) 131.

[39] Monceau, P., Peyrard, J., Richard, J. and Molinie, P., Phys. Rev. Lett. 39 (1977) 161

[40] Creuset, F., Creuzet, G. and Jerome, D., J. Phys. Lett. 46 (1985) 1079. 